\title{
THE UNION OF COMPACT SUBGROUPS OF AN ANALYTIC GROUP
}

\author{
TA-SUN WU
}

Dedicated to S. S. Koh

\begin{abstract}
Let $G$ be an analytic group. Let $\Omega(G)$ be the union of all compact subgroups of $G$. We give a necessary and sufficient condition for $\Omega(G)$ to be dense in $G$ in terms of the action of a maximal compact torus $T$ of $G$ on the nilradical $N$ of $G$.
\end{abstract}

Let $F$ be a locally compact group. Let $\Omega(F)$ be the union of all compact subgroups of $F$. We study the problem: when $\Omega(F)$ is dense in $F$. If $F$ is not connected, the problem is too broad to have any meaningful answers. On the other hand, if $F$ is almost connected, i.e., $F / F_{0}$ is compact where $F_{0}$ is the identity component of $F$, then the problem is quickly reduced to the case where $F$ is a Lie group with finitely many components. This is so because an almost connected locally compact $F$ has a maximal compact normal subgroup $M$ so that $F / M$ is a Lie group with finitely many components. It is easy to see that $\Omega(F)$ is dense in $F$ if and only if $\Omega(F / M)$ is dense in $F / M$. Let $G=F / M$. Let $G_{0}$ be the identity component of $G$. Since the identity component $G_{0}$ of $G$ is an open subgroup, so $\Omega(G) \cap G_{0}$ is dense in $G_{0}$ when $\Omega(G)$ is dense in $G$ (the converse is also true, cf. Theorem 2.10). Therefore, for most of this note we shall assume that $G$ is an analytic group. Now, let $G$ be an analytic group with $\Omega(G)$ dense in $G$. Let $M$ be the maximal compact normal subgroup of $G$. Again, $\Omega(G)$ is dense in $G$ if and only if $\Omega(G / M)$ is dense in $G / M$, so we may assume that $M$ is trivial. Let $N$ be the nilradical of $G$, i.e., the maximal analytic nilpotent normal subgroup of $G$. Then $N$ is simply connected since $M$ is trivial. Furthermore, by an argument due to Djokovic [1] we can show that $N$ is uniform in $G$. This implies that $G$ is a semidirect product $N \cdot K$ with $K$ a compact analytic group. Hence $K$ acts on $N$ as a group of automorphisms. The purpose of the present note is to show the following statement.

Theorem 2.7. Let $G$ be a semidirect product $N \cdot K$ with $N$ a simply connected analytic nilpotent group and $K$ a compact analytic group. Let $T$ be a maximal torus of $K$. Then $\Omega(G)$ is dense in $G$ if and only if the only element in $N$ fixed by $T$ is the identity element.

Another characterization of $\Omega(G)$ being dense in $G$ is the following condition.

Received by the editors October 23, 1989 and, in revised form, March 29, 1990.

1980 Mathematics Subject Classification (1985 Revision). Primary 22A05. 
Theorem 2.8. Let $G$ be an analytic group. Then the closure of $\Omega(G)$ has nonvoid interior if and only if $\Omega(G)$ is dense in $G$.

The present study was partially motivated by the well-known result in algebraic groups which says the union of all the Cartan subgroups of an irreducible algebraic group $G$ over an algebraic closed field of characteristic zero is an open and dense subset of $G$. For previous work on this subject, we refer to [1] and the references therein.

I am grateful to the referee who suggested I use results from algebraic groups (Lemma 2.4) to prove the main theorem (Theorem 2.7). This approach is much more elegant and clearer than my original elementary and lengthy proof (which implicitly duplicates arguments from algebraic group theory).

\section{Preliminary}

Let $F$ be an almost connected locally compact group. It is well known that $F$ has a maximal compact normal subgroup $M$ and $F / M$ is a Lie group with finite components. Let $G=F / M$ and $\pi$ be the canonical homomorphism from $F$ onto $G$. Let $\Omega(G)$ and $\Omega(F)$ be the union of compact subgroups of $G$ and $F$ respectively. Then $\Omega(F)=\pi^{-1}(\Omega(G))$. It is obvious that $\Omega(F)$ is a group if and only if $\Omega(G)$ is a group.

1.1. Lemma. Let $G$ be a Lie group with finitely many components. If the union $\Omega(G)$ of compact subgroups of $G$ is a group, then $\Omega(G)$ is the maximal compact normal subgroup of $G$.

Proof. Let $H$ be the closure of $\Omega(G)$. Since $\Omega(G)$ is a group, $H$ is a Lie group. Let $H_{0}$ be the identity component of $H$. Since $H_{0}$ is open in $H, H_{0} \Omega(G)$ is open (hence closed) and dense in $H$. Therefore, $H=H_{0} \Omega(G)$ is a dense subgroup of $H_{0}$. Every compact subgroup of $H_{0}$ is also a compact subgroup of $G$, so the union of all compact subgroups of $H_{0}$ is exactly $H_{0} \cap \Omega(G)$, i.e. $\Omega\left(H_{0}\right)=H_{0} \cap \Omega\left(G_{0}\right)$. In other words, $H_{0}$ is an analytic group with $\Omega\left(H_{0}\right)$ dense in $H_{0}$. Let $R S$ be a Levi decomposition of $H_{0}$ with $R$ the radical of $H_{0}$ and $S$ a maximal semisimple group. Let $N$ be the nilradical of $H_{0}$. Since $\Omega\left(H_{0}\right)$ is dense in $H_{0}$, so $\Omega\left(H_{0} / N\right)$ is dense in $H_{0} / N$. This implies that $H_{0} / N$ is compact. (Cf. Proposition 2.1 [1] or the proof of Theorem 2.8 of this article.)

Now, for simplicity, we first assume that $N$ is simply connected. Then $H_{0}=$ $K \cdot N$ (semidirect product) with $K$ a maximal compact subgroup of $H_{0}$. Let $n$ be any element of $N$ and $k$ be any element of $K$. Then $n k n^{-1} \in \Omega\left(H_{0}\right)$. Since $\Omega\left(H_{0}\right)$ is a group, so $n k n^{-1} k^{-1}$ is a compact element. But $n k n^{-1} k^{-1} \in N$. Therefore $n k n^{-1} k^{-1}$ is the identity of $H_{0}$. This implies that $H_{0}$ is in fact a direct product $K \times N$. Because $\Omega\left(H_{0}\right)$ is dense in $H_{0}, N$ must be trivial and $H_{0}$ is a compact analytic group.

Now, in case $N$ is not simply connected, $N$ has a maximal compact normal subgroup $Q$ such that $N / Q$ is simply connected. $Q$ is a characteristic subgroup of $H_{0}$. From the above argument, $H_{0} / Q$ is compact. Therefore $H_{0}$ is a compact analytic group.

Now $H=H_{0} \Omega(G)$. By Theorem 2 of [1], $H / H_{0}$ is compact. Hence $\Omega(G)$ is a compact subgroup of $G$.

What remains to be shown is that $\Omega(G)$ is normal in $G$. Since $\Omega(G)=$ $\bigcup_{g \in G} g Q g^{-1}$ with $Q$ a maximal compact subgroup of $G, \Omega(G)$ is $G$-invariant, 
i.e. $g \Omega(G) g^{-1}=\Omega(G)$. Therefore $\Omega(G)$ is the maximal compact normal subgroup of $G$. The proof of the lemma is complete.

1.2. Proposition. Let $F$ be an almost connected locally compact group. If $\Omega(F)$ is a group, then $\Omega(F)$ is the maximal normal subgroup of $F$.

Proof. Let $M$ be the maximal compact normal subgroup of $F$. Let $G=F / M$ and $\pi$ be the quotient map from $F$ onto $G$. The assertion follows immediately from Lemma 1.1 and the relation $\Omega(F)=\pi^{-1}(\Omega(G))$.

Now, we assume $\overline{\Omega(F)}$ is a group where $F$ is an almost connected group. Let $H=\overline{\Omega(F)}$. In general $H$ is not compact. However, it has the following interesting property.

1.3. Proposition. Let $F$ be an almost connected locally compact group. Let $H=\Omega(F)^{-}$. If $H$ is a group, then $H$ is almost connected and $\Omega(H)$ is dense in $H$.

Proof. Let $M$ be the maximal compact normal subgroup of $F$. Let $G=F / M$. $G$ is a Lie group with finitely many components. Let $\pi$ be the quotient map from $F$ onto $G$. Then $H=\pi^{-1} \pi(H)$, since $M \subset H$. And $\pi(H)=\overline{\Omega(G)}$, $\overline{\Omega(G)}$ is a group. Since $\Omega(G)$ is $G$-invariant (under the conjugation), $\overline{\Omega(G)}$ is a closed normal subgroup of $G$. Let $E=\overline{\Omega(G)}$. Then $E=\Omega(G) E_{0}$. Since $E_{0}$ is a characteristic subgroup of $E, E_{0}$ is normal in $G$. We are going to show that $E$ has only finitely many components. Let $E_{1}=E \cap$ $G_{0}=\Omega(G) E_{0} \cap G_{0}$. Then $E_{1} / E_{0}$ is a discrete normal subgroup of $G_{0} / E_{0}$. So $E_{1} / E_{0}$ is central in $G_{0} / E_{0}$. Since every element in $E_{1} / E_{0}$ has a representative from $\Omega(G), E_{1} / E_{0}$ is a torsion central subgroup of $G_{0} / E_{0}$. Hence $E_{1} / E_{0}$ is finite. Since $E G_{0} / G_{0} \cong E / E \cap G_{0}$, and $G$ has only finitely many components, therefore $E / E_{1}$ is finite. We conclude that $E$ is a Lie group with finitely many components. Let $Q$ be a maximal compact subgroup of $E$. By a result of G. D. Mostow, such a subgroup exists and any two of them are conjugate by an inner automorphism. Since $E=\overline{\Omega(G)}, Q$ is also a maximal compact subgroup of $G . \Omega(G)=\bigcup_{g \in G} g Q g^{-1}=\bigcup_{g \in E} g Q g^{-1}$ the last equality follows from the fact: $g Q g^{-1}$ is a maximal compact subgroup of $E$. Hence $E=\overline{\Omega(G)}=\overline{\Omega(E)}$.

Finally, $H=\overline{\Omega(F)}=\pi^{-1} \overline{\Omega(G)}=\pi^{-1}(E)$. Hence $H$ is almost connected and $\overline{\Omega(H)}=H$ as desired. Now, the proof is complete.

In view of Proposition 1.3, the problem of when $\overline{\Omega(F)}$ is a group can be reduced to the following situation.

1.4. Proposition. Let $F$ be a Lie group with finitely many components. Let $M$ be the maximal compact normal subgroup of $F$. Let $G=F / M$. If $\Omega(F)$ is dense in $F$, then $G=N \cdot Q$ a semidirect product with $N$ a simply connected nilpotent normal analytic subgroup and $Q$ a compact Lie group. Furthermore $Q$ can be identified faithfully as a group of automorphisms of $N$.

Proof. Since $G$ has no nontrivial compact normal subgroup, the nilradical $N$ of $G$ is a simply connected analytic group. Let $R$ be the radical of $G$ and $S$ be a semisimple Levi factor of $G$. Since $\Omega(G)$ is dense in $G$, so is $\Omega(G / R)$ dense in $G / R$. The group $G / R$ is a finite extension of the semisimple group $S$. Let us first remark if $E$ is a Lie group with finitely many components and $\overline{\Omega(E)}=E$, then $\overline{\Omega\left(E_{0}\right)}=E_{0}$ since $E_{0}$ is a open normal subgroup of $E$. If $S$ 
has nontrivial noncompact simple factor $S^{\prime}, \Omega(S)$ cannot be dense in $S^{\prime}$ by the structure of $S^{\prime}$. (Cf. Proposition 2.1 of [1] or the proof of Theorem 2.8 in this article.) Therefore $S$ must be compact. Note that $N S$ is a closed normal subgroup of $G, G / N S$ is a finite extension of an analytic abelian group $A$. If the vector part of $A$ is nontrivial then $\Omega(G / N S)$ cannot be dense in $G / N S$. Hence we conclude that $G / N$ must be a compact group, a fortiori, $G=N \cdot Q$. Let $\rho$ be the homomorphism from $Q$ into the group of automorphisms of $N$ defined by $\rho(q)=\phi_{q}$ where $\phi_{q}: N \rightarrow N, \phi_{q}(n)=q n q^{-1}$. Since the kernel $\rho$ is a compact normal subgroup of $G$, it is a trivial group. Hence $\rho$ is a faithful representation, i.e. $Q$ can be identified as a group of automorphisms of $N$.

\section{ANALYTIC GROUPS WITH DENSE SUBSETS OF COMPACT ELEMENTS}

Let $F$ be a locally compact almost connected group. Let $M$ be the maximal compact normal subgroup of $F$. By Proposition 1.5, $\Omega(F)$ is dense in $F$ if and only if $\Omega(F / M)$ is dense in $F / M$. Let $G^{\prime}=F / M$. When $\Omega(F)^{-}=F$, then $G^{\prime}=N \cdot Q$ where $N$ is a simply connected nilpotent analytic normal subgroup of $G^{\prime}$ and $Q$ is a maximal compact Lie group which can be identified as a group of automorphisms of $N$. Let $K$ be the identity component of $Q$. Then $G=N \cdot K$ is the identity component of $G^{\prime}$. In this section, we shall show that $\Omega\left(G^{\prime}\right)$ is dense in $G^{\prime}$ if and only if $\Omega(G)$ is dense in $G$. We first give a necessary and sufficient condition for $\Omega(G)$ to be dense in $G$.

From now on we shall always assume that $G=N \cdot K$ a semidirect product with $N$ a simply connected nilpotent analytic group and $K$ an analytic compact group. Furthermore, $K$ can be faithfully represented as a subgroup of automorphisms of $N$. With this identification, sometimes it is convenient to write $k(n)$ instead of $k n k^{-1}$ for $k \in K, n \in N$. We shall do so in the future.

We shall always use $T$ to denote a maximal torus of $K$. Let $W$ be the normalizer of $T$ in $K, W=\mathscr{N}_{k}(T)$. Then $W / T$ is finite. Let $x$ be an element of $G$. We say $x$ is a compact element if the smallest closed subgroup of $G$ containing $x$ is compact, i.e. $\overline{\langle x\rangle}$ is compact where $\langle x\rangle=\left\{x^{n}: n\right.$ integer $\}$. An element $t$ of $T$ is a dense element in $T$ if $\overline{\langle t\rangle}=T$.

Since all the maximal compact subgroups of $G$ are conjugate by inner automorphisms induced by elements from $N$, the following statement is obvious.

2.1. Lemma. $\Omega(G)=\bigcup_{g \in G} g K g^{-1}=\left\{\left(n k\left(n^{-1}\right), k\right): n \in N, k \in K\right\}$.

2.2. Lemma. If $\Omega(N \cdot T)$ is dense in $N \cdot T$, then $\Omega(G)$ is dense in $G$.

Proof. Given any element $(n, k)$ of $N \cdot K, k=x t x^{-1}$ for some $t$ in $T$ and $x$ in $K$. Since $\Omega(N \cdot T)$ is dense in $N \cdot T$, there exists a sequence of compact elements $\left(n_{i} t_{i}\left(n_{i}^{-1}\right), t_{i}\right)$ in $N \cdot T$ which converges to $\left(x^{-1}(n), t\right)$. Then

$$
\begin{aligned}
\lim (1, x)\left(n_{i} t_{i}\left(n_{i}^{-1}\right), t_{i}\right)(1, x)^{-1} & \\
& =(1, x)\left(x^{-1}(n), t\right)(1, x)^{-1} \\
& =\left(n, x t x^{-1}\right)=(n, k), \quad(n, k) \in \overline{\Omega(N \cdot K)} .
\end{aligned}
$$

2.3. Lemma. If $\Omega(G)$ is dense in $G$, then $N \cdot T=\overline{\Omega(N \cdot T)}{ }^{W}$ (notation: $x$ and $y$ are elements of $\left.G, x^{y}=y x y^{-1}\right)$.

Proof. Let $t$ be any dense element of $T$. Let $n$ be any element of $N$. Since $\Omega(G)$ is dense in $G,(n, t)$ is the limit of a sequence of compact elements 
$\left(n_{i} k_{i}\left(n_{i}^{-1}\right), k_{i}\right)$ in $\Omega(N \cdot K)$. Express $k_{i}=y_{i} t_{i} y_{i}^{-1}$ with $y_{i}$ from $K$ and $t_{i}$ from $T$. Then $t=\lim k_{i}=\lim y_{i} t_{i} y_{i}^{-1}$. Since $K$ is compact, taking a subsequence, we may assume that $y=\lim y_{i}, t^{\prime}=\lim t_{i}$. Hence $t=y t^{\prime} y^{-1}$. Because $t$ is a dense element of $T$, so $t^{\prime}$ is also a dense element of $T$, a fortiori, $y$ leaves $T$ invariant, $y$ is in the normalizer $W$ of $T$. Now,

$$
\left(y^{-1}(n), t^{\prime}\right)=\lim \left(y_{i}^{-1}\left(n_{i}\right) t_{i}^{-1}\left(y_{i}\left(n_{i}^{-1}\right)\right), t_{i}\right) \in \overline{\Omega(N \cdot T)} .
$$

Hence $(n, t)=(1, y)\left(y^{-1}(n), t^{\prime}\right)(1, y)^{-1} \in \overline{\Omega(N \cdot T)}^{W}$. The proof is now complete.

It is clear that $G=N \cdot K$ can be embedded as a closed subgroup of some general linear group $\mathrm{GL}(n, \mathbb{R})$ with $N$ as its unipotent radical. Let us identify $G$ with its image. So $G$ is an algebraic group over the real number field, and we can apply results from the theory of algebraic groups. Since $K$ is a compact analytic group, every element in $K$ is a semisimple element. For each $k \in K$, define the function $\theta_{k}: N \rightarrow N$ by $\theta_{k}(n)=k(n) n^{-1}$. Let $N^{k}=\{n \in N: k(n)=n\}$. Let $C(k, N)=\theta_{k}(N)=\left\{k(n) n^{-1}: n \in N\right\}$. The results we need from algebraic groups is the following statement.

2.4. Lemma. Let $k \in K$. We have the following conditions.

(i) $N^{k}$ and $C(k, N)$ are algebraic varieties over the real number field. The product map from $N^{k} \times C(k, N) \rightarrow N$ is a surjective isomorphism of varieties. Furthermore: $\theta_{k}: C(k, N) \rightarrow C(k, N)$ is a rational automorphism of $C(k, N)$.

(ii) The element $(n, k) \in N \cdot K$ is a compact element if and only if $n \in$ $C(k, N)$.

Proof. The statement (i) is a special case of a general result due to Borel and Tits (Lemma 11.1 of [3]). So we show (ii). Since $K$ is a maximal compact subgroup of $N \cdot K,(n, k)$ is a compact element if and only if there exists an element $n_{0}$ of $N$ such that

$$
\left(n_{0}, 1\right)(n, k)\left(n_{0}, 1\right)^{-1} \in\{1\} \cdot K .
$$

Equivalently, $\left(n_{0} n k\left(n_{0}^{-1}\right), k\right)=(1, k)$. Hence $(n, k)$ is compact if and only if $n=n_{0}^{-1} k\left(n_{0}\right) \in C(k, N)$.

2.5. Lemma. For each $t \in T, \theta_{t^{2}}=\theta_{t}^{2}, C\left(t^{2}, N\right) \subset C(t, N)$. Let $t_{0}$ be a dense element of $T$. Then $C(t, N) \subset C\left(t_{0}, N\right)$ for any $t \in T$.

Proof. The fact $\theta_{t^{2}}=\theta_{t}^{2}$ follows from a simple computation. Hence $C\left(t^{2}, N\right)$ $\subset C(t, N)$. Let $t_{0}$ be a dense element. Then $t=\lim t_{0}^{l_{i}}$ for some sequence $t_{0}^{l_{i}}$. Hence $\theta_{t}(n)=\lim \theta_{t_{0}}^{l_{i}}(n) \in C\left(t_{0}, N\right)$ since $C\left(t_{0}, N\right)$ is topologically closed, a fortiori, $C(t, N) \subset C\left(t_{0}, N\right)$.

2.6. Lemma. Let $t_{0} \in T$ such that $C\left(t_{0}, N\right)=N$. Then there exists a neighborhood $V$ of $t_{0}$ in $K$ such that $C(t, N)=N$ for any $t \in K$.

Proof. Let $Z$ be the center of $N$. Then $Z$ is $T$-invariant. If $Z=N$, then $\theta_{t_{0}}=I-\operatorname{Ad} t_{0}$ is just a linear transformation of $Z$. If $\theta_{t_{0}}$ is surjective, then there exists a neighborhood $V$ of $t_{0}$ in $K$ so that $\theta_{t}=I-\operatorname{Ad} t$ is surjective for $t \in V$. Now, we assume the lemma has been proved for nilpotent groups with shorter length of upper central series of $N$. Since $N^{t_{0}}=\{1\}$, so the 
restriction of $t_{0}$ on $Z$ has no nontrivial fixed element. Therefore, there exists a neighborhood $V_{1}$ of $t_{0}$ in $K$ such that $\theta_{t}: Z \rightarrow Z$ is surjective for each $t \in V_{1}$. On the other hand, $\theta_{t_{0}}$ is clearly surjective on $N^{\prime}=N / Z$, therefore, there is a neighborhood $V_{2}$ of $t_{0}$ in $K$ such that $\theta_{t}$ is surjective on $N^{\prime}$. Let $V=V_{1} \cap V_{2}$ and let $t \in V$ and $n \in N$. Since $t \in V_{2}$, there exists an element $n^{\prime} \in N$ such that $\theta_{t}\left(n^{\prime}\right)=n z$ for some $z \in Z$. There exists an element $z^{\prime} \in Z$ such that $\theta_{t}\left(z^{\prime}\right)=z^{-1}$. Then $\theta_{t}\left(n^{\prime} z^{\prime}\right)=\theta_{t}\left(n^{\prime}\right)\left(\theta_{t}\left(z^{\prime}\right)\right)=n$. Now the proof is complete.

2.7. Theorem. Let $G=N \cdot K$ a semidirect product with $N$ a simply connected analytic nilpotent group and $K$ a compact analytic group. Let $T$ be a maximal torus of $K$. Let $\Omega(G)$ be the union of all compact subgroups of $G$. Then $\Omega(G)$ is dense in $G$ if and only if $T$ does not leave any element in $N$ fixed except the identity of $N$. Furthermore, when $\Omega(G)$ is dense in $G, \Omega(G)$ contains a dense open subset of $G$.

Proof. Let $N^{T}=\bigcap_{t \in T} N^{t}$, the set of $T$-fixed elements in $N$. Let $t_{0}$ be a dense element of $T$. Then $N^{T}=N^{t_{0}}$.

(I) Assume that $N^{T}$ is nontrivial, i.e. $N^{T} \neq\{1\}$. By Lemma 2.6 and (ii) of Lemma 5, $\Omega(N \cdot T) \subset C\left(t_{0}, N\right) \cdot T$. Since $C\left(t_{0}, N\right) \cdot T$ is closed, $\overline{\Omega(N \cdot T)} \subset$ $C\left(t_{0}, N\right) \cdot T$. Since $W / T$ is finite, $\left(C\left(t_{0}, N\right) \cdot T\right)^{W}$ is properly contained in $N \cdot T$ if $N^{t_{0}}$ is nontrivial. Therefore $\Omega(G)$ is not dense in $G$ by Lemma 2.4.

(II) Assume that $N^{T}=\{1\}$. Let $D$ be the set of dense elements of $T$. Then $N^{T}=N^{d}=\{1\}$ and $C(d, N)=N$ for every $d \in D$. By (ii) of Lemma 2.5, $N \cdot D \subset \Omega(N \cdot T)$. Since $N \cdot D$ is dense in $N \cdot T, \Omega(N \cdot T)$ is dense in $N \cdot T$. By Lemma 2.3, $\Omega(G)$ is dense in $G$. Finally, if $\Omega(G)$ is dense in $G$, then interior of $\Omega(G)$ is dense in $G$ by Lemma 2.6. Now the proof is complete.

2.8. Theorem. Let $G$ be an analytic group. Then $\Omega(G)$ is dense in $G$ if and only if the closure of $\Omega(G)$ has nonvoid interior.

Proof. If $\Omega(G)$ is dense in $G$, trivially, the closure of $\Omega(G)$ has nonvoid interior. So, we show if the closure of $\Omega(G)$ has nonvoid interior then $\Omega(G)$ is dense in $G$. Clearly we may assume that $G$ has no nontrivial compact normal subgroup. Now, if $\Omega(G)^{-}$has nonvoid interior, then every quotient group of $G$ also enjoys the same property. Hence the quotient of its noncompact semisimple factor $S$ also has the same property. Recall all the eigenvalues of the compact elements have module one (when we consider the adjoint representation). By assumption there exists an open set in $S$ such that every element has all its eigenvalues of module one. This is clearly impossible (in view of Iwasawa decomposition $S=\mathrm{KAN}$ ) unless $S$ is a compact group. Hence $G$ has no noncompact simple factor. Then $G$ is faithfully linear representable (since it has no nontrivial compact normal subgroup). Thus the commutator $[G, G]$ is closed and $G /[G, G]$ is an abelian analytic group. It is clear the closure of $\Omega(G /[G, G])$ cannot have nonvoid interior unless $G /[G, G]$ is compact by the structure of abelian analytic group. Therefore we conclude $G=N \cdot K$ with $N$ a simply connected nilpotent normal analytic group and $K$ a compact analytic group. Let $L=$ int $\Omega(G)^{-}$, interior of the closure of $\Omega(G)$. Then $L \cap N \cdot T$ is a nonvoid open subset of $N \cdot T$ where $T$ is a maximal torus of $G$. Now, suppose $\Omega(G)$ is not dense in $G$, then $\Omega(N \cdot T)$ is not dense in $N \cdot T$ by Lemma 2.2. Hence there exists a dense point $t$ in $T$ such that $N^{t}$ is nontrivial and 
$\Omega(N \cdot T) \subset C(t, N) \times T$. This shows that $L \cap N \cdot T$ is not open in $N \cdot T$. We have a contradiction. Therefore we conclude that $\Omega(G)$ is dense in $G$. And the proof is now complete.

Previously, we have concentrated our discussions on analytic groups. Now, we shall study Lie groups with finitely many connected components.

In view of Proposition 1.4, we may assume that $G^{\prime}=N \cdot Q$ where $N$ is a simply connected normal nilpotent analytic group and $Q$ is a compact Lie group. Let $K$ be the identity component of $Q$. Let $G=N \cdot K$. Let $K=Q \cap$ $G_{0}$. Then $K$ is a maximal compact subgroup of $G$. It is known that there exists a finite subgroup $D$ of $Q$ such that $Q=K D$ (cf. the appendix).

We reserve all the notations in the following discussions.

2.9. Lemma. Let $d \in D$. Let $\langle d\rangle$ denote the cyclic group generated by $d$. Then $\Omega(G) \cap\langle d\rangle G=\Omega(\langle d\rangle G)$.

Proof. Since $\Omega(G)=\bigcup_{x \in G} x Q x^{-1}$. Let $q \in Q$ and $x \in G$. If $x q x^{-1} \in d^{l} G$, then $q \in d^{l} G \subset\langle d\rangle G$. Hence $x q x^{-1} \in \Omega(\langle d\rangle G)$.

2.10. Theorem. Let $G^{\prime}$ be a Lie group with finitely many components. Let $G$ be the identity component of $G^{\prime}$. Then $\Omega\left(G^{\prime}\right)$ is dense in $G^{\prime}$ if and only if $\Omega(G)$ is dense in $G$.

Proof. In essence, we only need to show $\Omega\left(G^{\prime}\right)^{-}=G^{\prime}$ when $\Omega(G)^{-}=G$. For this purpose, we may write $G^{\prime}=N \cdot K D$ with $N$ a simply connected nilpotent analytic group, $K$ a compact analytic group, and $D$ a finite group which normalizes $K$. By Lemma 2.9 , we may assume that $D=\langle d\rangle$, a finite cyclic group. We assume that $\Omega(G)$ is dense in $G$.

Now, we assume that $N$ is an abelian group first. Given any $k \in K$, then $k$ belongs to some maximal torus of $K$, say $T$. Since $\overline{\Omega(G)}=G, T$ acts on $N$ without any nontrivial fixed points. There exists a residue subset of dense elements $t$ of $T$ arbitrarily close to $k$ such that $d^{-1}-t$ is a surjection. Suppose this is not true, i.e., $d^{-1}(n)-t(n)=0$. Because $d^{-1}(n)=t(n)$, $\left(d^{-1}\right)^{l}(n)=t^{l}(n)$ for any integer $l$. Since $d$ is a torsion element, so $\left(d^{-1}\right)^{l}=\mathrm{id}$ for some positive integer $l$. Then we have $t^{l}(n)=n$. Since $t^{l}$ is also a dense element, it does not have any nontrivial fixed vector, we reach a contradiction. Hence $\left(d^{-1}-t\right)(N)=N, d^{-1}-t$ is a surjection. Now, let $n$ be any element in $N$, there is an element $m$ such that $\left(d^{-1}-t\right)(m)=d^{-1}(n)$, equivalently, $d\left(d^{-1}-t\right)(m)=n$. From this we have

$$
(m, 1)(1, d t)(m, 1)^{-1}=(m-d t(m), d t)=\left(d\left(d^{-1}-t\right)(m), d t\right)=(n, d t) .
$$

Hence $d G=\left(\Omega\left(G^{\prime}\right) \cap d G\right)^{-}$.

Now, we use the induction on the length of central series of $N$ to show that $\Omega\left(G^{\prime}\right)$ is dense in $G^{\prime}$. Assume $N^{(l)}$ is central in $N$ and $\Omega\left(G^{\prime} / N^{(l)}\right)$ is dense in $G^{\prime} / N^{(l)}$. Let $k$ be an element of the maximal torus $T$, and $U$ a neighborhood of $k$ in $T$. There exists a residue subset $S$ of dense elements of $T$ such that (1) $S$ is dense in $U$ (2) for each $s \in S$ and $m \in N / N^{(l)}$ there is an element $x$ in $N / N^{(l)}$ such that $x d s\left(x^{-1}\right)=m$. Let $\pi$ be the quotient map from $G^{\prime}$ onto $G^{\prime} / N^{(l)}$. Let $n$ be any element in $N$. Let $m=\pi(n)$. Then we have $x d s\left(x^{-1}\right)=n z$ for some $z$ in $N^{(l)}$. Since $N^{(l)}$ is central in $N$, it is an abelian group. We know there exists a residue subset $S^{\prime}$ of $U$ such that (1) $S^{\prime}$ is dense 
in $U$ (2) $S^{\prime}$ consists of dense elements of $T$. And for each $z \in N^{(l)}$, there exists $z^{\prime}$ in $N^{(l)}$ with the property: $z^{\prime} d s^{\prime}\left(z^{\prime-1}\right)=z^{-1}$. Now, choosing any $s \in S \cap S^{\prime}$, we have

$$
x z^{\prime} d s\left(z^{\prime-1} x^{-1}\right)=n .
$$

And the proof is now complete.

2.11. Examples. We have expressed the condition for the density of $\Omega(G)$ in terms of the actions of its maximal torus $T$ on the nilradical $N$ of $G$. The following example illustrates the usefulness of such criteria:

Let $G$ be the subgroup of $\mathrm{GL}(4, \mathbb{R})$ which consists of the following matrices

$$
\left(\begin{array}{llll} 
& & & x \\
& A & & y \\
& & & z \\
0 & 0 & 0 & 1
\end{array}\right)
$$

where $A \in \mathrm{SO}(3, \mathbb{R})$ and $x, y, z$ are real numbers. Then $G \cong \mathbb{R}^{3} \cdot \mathrm{SO}(3, \mathbb{R})$ with $N \cong \mathbb{R}^{3}$ and $K \cong \mathrm{SO}(3, \mathbb{R})$. The maximal torus $T$ of $K$ is a circle group. Since dimension of $N$ is an odd number, $T$ does not act effectively on $N$. Therefore $\Omega(G)$ is not dense in $G$. Observe $\operatorname{SO}(3, \mathbb{R})$ acts on $N$ without any nontrivial fixed element.

Now, replace $\mathrm{GL}(4, \mathbb{R})$ by $\mathrm{GL}(5, \mathbb{R})$ and $\mathrm{SO}(3, \mathbb{R})$ by $\mathrm{SO}(4, \mathbb{R})$ in the above construction of $G$, i.e. $G \cong \mathbb{R}^{4} \cdot S(4, \mathbb{R})$, then $\Omega(G)$ is dense in $G$ since the maximal torus acts effectively on $N$.

2.12. Proposition. Let $G$ be an analytic group, let $H$ be a subgroup of $\Omega(G)$ and let $F=H^{-}$. If $F_{0}$ is solvable then $F_{0}$ is compact and $F \subset \Omega(G)$.

Proof. Since $F$ is a Lie group, $F_{0}$ is an open subgroup of $F$. Hence $F=F_{0} H$ and $H \cap F_{0}$ is a dense subgroup of $F_{0}$. Since $H \cap F_{0} \subset \Omega(G),\left(H \cap F_{0}\right)^{-}$is compact by Theorem 5.2 of [2], a fortiori, $F_{0} \subset \Omega(G)$. Therefore $F=H^{-}=$ $F_{0} H \subset \Omega(G)$.

Now, we consider a closed analytic subgroup $H$ of a semisimple analytic group $G$ which is contained in $\overline{\Omega(G)}$. First, we need the following lemma.

2.13. Lemma. Every unipotent element $x$ of $\operatorname{SL}(2, \mathbb{R})$ is in the closure of the union of all compact subgroups of $\operatorname{SL}(2, \mathbb{R}) ; x \in \Omega(\operatorname{SL}(2, \mathbb{R}))$.

Proof. Every unipotent element $x$ of $\operatorname{SL}(2, \mathbb{R})$ is conjugate to a matrix of the following form

$$
A=\left(\begin{array}{ll}
1 & \gamma \\
0 & 1
\end{array}\right) .
$$

To show $x \in \overline{\Omega(\operatorname{SL}(2, \mathbb{R}))}$, we only need to show $A \in \overline{\Omega(\operatorname{SL}(2, \mathbb{R}))}$. First, assume $\gamma$ is positive. Then $\gamma=\delta^{2}, \delta>0$.

By computation

$$
\begin{aligned}
\left(\begin{array}{ll}
1 & \gamma \\
0 & 1
\end{array}\right)= & \lim _{n \rightarrow \infty}\left(\begin{array}{cc}
\delta n & \delta n \\
1 / \delta n & 2 / \delta n
\end{array}\right) \\
& \cdot\left(\begin{array}{cc}
\left(1-1 / n^{4}\right)^{1 / 2} & 1 / n^{2} \\
1 / n^{2} & \left(1-1 / n^{4}\right)^{1 / 2}
\end{array}\right)\left(\begin{array}{cc}
2 / \delta_{n} & -\delta n \\
-1 / \delta n & \delta n
\end{array}\right) .
\end{aligned}
$$

Hence $A \in \overline{\Omega(\operatorname{SL}(2, \mathbb{R}))}$. When $\gamma$ is a negative number, $A$ is the inverse of $\left(\begin{array}{cc}1 & -\gamma \\ 0 & 1\end{array}\right)$, hence $A \in \overline{\Omega(\operatorname{SL}(2, \mathbb{R}))}$. 
2.14. Remark. One may consider the matrix $B=\left(\begin{array}{ll}a & b \\ 0 & 1\end{array}\right)$ in $\operatorname{SL}(2, \mathbb{R})$. The characteristic polynomial of $B$ is $\lambda^{2}-(a+d) \lambda+1$. When $(a+d)^{2}<4$, the eigenvalues of $B$ have absolute values $1, B$ is an elliptic element (compact element). It is easy to see we can find compact elements which approximate to a unipotent matrix $\left(\begin{array}{ll}1 & \gamma \\ 0 & 1\end{array}\right)$ since we can choose suitable numbers for $b$ and $c$.

2.15. Proposition. Let $G$ be a linear semisimple analytic group. Then every unipotent element of $G$ is in the closure of $\Omega(G)$.

Proof. It is known that every unipotent element $x$ in $G$ belongs to an analytic group which is locally isomorphic with $\operatorname{SL}(2, \mathbb{R})$. By Lemma 2.13, $x \in \overline{\Omega(G)}$.

2.16. Corollary. Let $G$ be a linear semisimple analytic group. Let $G=\mathrm{KAN}$ an Iwasawa decomposition. Then every closed analytic subgroup $H$ of $G$ contained in $\Omega(G)$ is conjugate to a subgroup $M \cdot Q$ (a semidirect product) with $M \subset N$ and $Q \subset K$. Conversely, every conjugate of $M \cdot Q$ is a subgroup in $\overline{\Omega(G)}$.

Proof. (1) Assume $H$ is conjugate to $M \cdot Q$ with $M \subset N$ and $Q \subset K$. By Proposition $12 M \cdot Q \subset \overline{\Omega(G)}$. Hence every conjugate of $M \cdot Q$ is also a subgroup of $\overline{\Omega(G)}$ since $\overline{\Omega(G)}$ is invariant under conjugation.

(2) Assume $H \subset \overline{\Omega(G)}$. Every element $h$ of $H$ has all its eigenvalues of absolute value 1 . Hence $H=E \cdot P$ where $E$ is the nilradical of $H$ consisting of unipotent elements and $P$ is a compact analytic group. Since the compact subgroup of $G$ is conjugate to a subgroup of $K$, we may assume that $P \subset K$. Since $E$ is a group of unipotent elements, it is known that $E$ is conjugate to a subgroup of $N$ by the conjugation defined by an element from $K$. Therefore, $H$ is conjugate to a subgroup $M \cdot Q$ with $M \subset N, Q \subset K$. The proof is now complete.

\section{APPENDIX}

Here, we shall give a shorter proof of the following useful statement originally due to D. H. Lee (Math. Z. 104 (1968), 28-49).

1. Proposition. Let $G$ be a Lie group with finitely many components. Then there exists a finite subgroup $D$ of $G$ such that $G=G_{0} D$.

Proof. Since $G$ has only finitely many components, by a result due to G. D. Mostow, $G=K E$ where $K$ is a maximal compact subgroup of $G$ and $E$ is a simply connected manifold. $E \subset G_{0}$. Hence, $G=K G_{0}$. Let $T$ be a maximal torus of the identity component $K_{0}$ of $K$. Let $S$ be the normalizer of $T$ in $K$. Then $S$ is a compact Lie group with its identity component $T$. Let $x$ be any element of $K$. Since $x T x^{-1}$ is also a maximal torus of $K$, there exists an element $y$ in $K_{0}$ such that $x T x^{-1}=y T y^{-1}$. Therefore $y^{-1} x \in S$. Hence $x \in S y \subset S K_{0}, K=S K_{0}, G=G_{0} K=G_{0} K_{0} S=G_{0} S$. Once we show there exists a finite subgroup $D$ of $S$ such that $S=T D$ we will have $G=G_{0} S=G_{0} T D=G_{0} D$.

In passing, we note the above method reduces our original problem to an extension problem: $1 \rightarrow T \rightarrow S \rightarrow S / T \rightarrow 1$ with an abelian divisible kernel $T$. So, we can apply a method from cohomology theory.

Let $X=S / T$ the left coset space. $X$ is a finite set. Let $r$ be the cardinality of $X$. Let $\sigma$ be a cross section of $S$ over $X$, i.e. $\sigma=X \rightarrow S$ such that 
$\pi \circ \sigma=$ id. Here $\pi$ is the canonical map from $S$ onto $S / T$. Define the function $\phi$ from $S$ into $T$ by the rule $\phi(s)=\prod_{x \in X} s \sigma(x) \sigma(s x)^{-1}$. Let $t \in T$. Then $\phi(t)=t^{r}$. Since $T$ is a divisible group $\phi(T)=T$. The function $\phi$ has following basic property

$$
\begin{aligned}
\phi\left(s_{1} s_{2}^{-1}\right) & =\prod_{x \in X} s_{1} s_{2}^{-1} \sigma(x) \sigma\left(s_{1} s_{2}^{-1} x\right)^{-1} \\
& =\prod_{x \in X} s_{1} s_{2}^{-1} \sigma(x) \sigma\left(s_{2}^{-1} x\right)^{-1} s_{1}^{-1} s_{1} \sigma\left(s_{2}^{-1} x\right) \sigma\left(s_{1} s_{2}^{-1} x\right)^{-1} \\
& =s_{1}\left\{\prod_{x \in X} s_{2}^{-1} \sigma(x) \sigma\left(s_{2}^{-1} x\right)^{-1}\right\} s_{1}^{-1} \prod_{x \in X} s_{1} \sigma\left(s_{2}^{-1} x\right) \sigma\left(s_{1} s_{2}^{-1}\right)^{-1} \\
& =s_{1} \phi\left(s_{2}^{-1}\right) s_{1}^{-1} \phi\left(s_{1}\right) .
\end{aligned}
$$

Now, let $D=\{s \in S: \phi(s)=1\}$. By the above equality, $D$ is a group. Let $s$ be any element of $S$. Since $\phi(T)=T$, there exists an element $t$ in $T$ such that $\phi(s)=\phi(t)$. Since $\phi\left(t^{-1} s\right)=t^{-1} \phi\left(s^{-1}\right) t \phi(t)=1$. Hence $t^{-1} s \in D, S=T D$. Then $D / D \cap T \approx S / T$ is a finite coset space. Since $D \cap T$ is the kernel of the homomorphism $\phi: T \rightarrow T, \phi(t)=t^{r}, D \cap T$ is a finite group. Hence $D$ is a finite group. Now the proof is complete.

Using the above proposition, we have the proof of the following theorem which was also due to D. H. Lee (in the same article cited above).

2. Theorem. Let $G$ be an almost connected locally compact group. Then there exists a compact totally disconnected subgroup $D$ of $G$ such that $G=G_{0} D$.

Proof. Since $G$ is almost connected, by a theorem of G. D. Mostow, $G=G_{0} K$ where $K$ is a maximal compact subgroup of $G$. Let $A$ be a maximal compact connected abelian subgroup of $K$. Let $B$ be the normalizer of $A$ in $K$. Since the maximal compact connected abelian subgroup of $K$ is conjugate by inner automorphisms defined by elements from $k_{0}, K=K_{0} B$. The identity component $B_{0}$ of $B$ is the compact connected abelian group $A$. We are going to show there exists a compact totally disconnected subgroup $D$ of $B$ such that $B=A D$. From this we shall have the statement $G=G_{0} D$.

Let $X$ be the left coset space $B / A . X$ is a compact totally disconnected space. It is known that there is a global cross section $\sigma: X \rightarrow B$. (Cf. K. H. Hofmann and P. S. Mostert, Elements of compact semigroups, Appendix II, Theorem 1.13, p. 318.) Let $\delta$ be the canonical homomorphism from $B$ onto $X$. Then $\delta \circ \sigma: X \rightarrow X$ is the identity map.

Since $B$ is the inverse limit of compact Lie groups with torus groups as its identity component; there exists an inverse system of compact normal subgroups $\left\{L_{\lambda}\right\}$ of $B$ such that $B=\lim B_{\lambda}, B_{\lambda}=B / L_{\lambda}$. Let $A_{\lambda}=A L_{\lambda} / L_{\lambda}$. Then $A=\lim A_{\lambda}$ and $A_{\lambda}$ is a torus group for all $\lambda$. Let $X_{\lambda}=B / A L_{\lambda}$. We have the following commutative diagram:

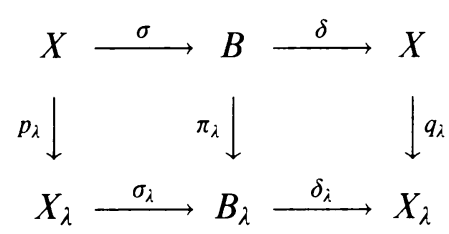


Here, $p_{\lambda}: X \rightarrow X_{\lambda}, \pi_{\lambda}: B \rightarrow B_{\lambda}$ are the canonical maps and $\sigma_{\lambda}$ is the cross section from $X_{\lambda}$ into $B_{\lambda}$ induced by $\sigma$. For each $\lambda$, define the function $\phi_{\lambda}: B_{\lambda} \rightarrow A_{\lambda}$ by the rule $\phi_{\lambda}\left(b_{\lambda}\right)=\prod_{x_{\lambda} \in X_{\lambda}} b_{\lambda} \sigma_{\lambda}\left(x_{\lambda}\right) \sigma_{\lambda}\left(b_{\lambda} x_{\lambda}\right)^{-1}$. Let $D_{\lambda}=\left\{b_{\lambda} \in\right.$ $\left.B_{\lambda} ; \phi_{\lambda}\left(b_{\lambda}\right)=1\right\}$. Then $B_{\lambda}=A_{\lambda} D_{\lambda}$ and $D_{\lambda}$ is a finite group by the above proposition. Let $\theta_{\lambda}: D_{\lambda} \rightarrow B_{\lambda}$ be the inclusion map, let $\theta: \lim D_{\lambda} \rightarrow \lim B_{\lambda}=$ $B$, and let $D=\theta\left(\underset{\lim }{\longleftarrow} D_{\lambda}\right)$. Then $D$ is compact and totally disconnected since $\lim D_{\lambda}$ is compact and totally disconnected. Moreover $B=A D$. Now, the proof is complete.

3. Remark. We may approach the proof of above theorem in the following way. For each $\lambda$ and every $b$ in $B$, the set $\bigcap_{\lambda} \pi_{\lambda}^{-1} \pi_{\lambda}^{-1}\left(\pi_{\lambda}(b)\right)$ consists of only one element of $A$, so we have a well-defined function $\phi: B \rightarrow A, \phi(b)=$ $\bigcap_{\lambda} \pi_{\lambda}^{-1} \phi_{\lambda}\left(p_{\lambda}(b)\right)$. The function $\phi$ satisfies $(*): \phi\left(b_{1} b_{2}\right)=b_{1} \phi\left(b_{2}\right) b_{1}^{-1} \phi\left(b_{1}\right)$. Let $D=\{b \in B ; \phi(b)=1\}$, then $D \cong \lim D_{\lambda} . \quad D$ is a compact totally disconnected subgroup of $B$ and $B=A D$. Thus $G=G_{0} D$ as desired.

Added in Proof. Professor D. Z. Djokovic kindly informed us that Theorem 2.10 has also been obtained by M. I. Kabenyuk, Connected groups with dense sets of compact elements, (Ukrainian Math. J. 33 (1981)).

\section{REFERENCES}

1. D. Z. Djokovic, The union of compact subgroups of a connected locally compact group, Math. Z. 158 (1978), 99-105.

2. S. P. Wang, Compactness properties of topological groups. II, Duke Math. J. 39 (1972), 243-251.

3. A. Bord and J. Tits, Groupes reductifs, Inst. Hautes Études Sci. Publ. Math. 27 (1965), $659-755$. 44106

Department of Mathematics, Case Western Reserve University, Cleveland, Ohio 